\title{
Stability studies of compression coated ornidazole tablets for colon specific drug delivery
}

\author{
Seda RENÇBER ${ }^{1}$, Zeynep ŞENYİĞİT ${ }^{2}$, Mine ÖZYAZICI ${ }^{1}$ * \\ ${ }^{1}$ Department of Pharmaceutical Technology, Faculty of Pharmacy, Ege University, Bornova, İzmir, Turkey \\ 2 Department of Pharmaceutical Technology, Faculty of Pharmacy, İzmir Katip Çelebi University, Çiğli, İzmir, Turkey \\ * Corresponding Author. E-mail: mine.ozyazici@ege.edu.tr (M.Ö.); Tel. 00902323111357; ORCID No: 0000-0002-3288- \\ 0685 .
}

Received: 22 May 2018 / Revised: 09 August 2018 / Accepted: 14 August 2018

\begin{abstract}
In our previous study, colon targeted tablets of ornidazole were prepared with compression coating technique. Pectin was used as the coating layer and different ethyl cellulose (EC) types (EC N7, N 10 and N 100) were added to Pectin to enhance mechanical and release properties. The object of the present study was performed stability testing of these coated tablets of ornidazole in a high density polyethylene plastic bottle with/without silica gel as per ICH guidelines. For this aim, several quality-control parameters such as uniformity of weight, diameter and thickness of tablets, tablet hardness, friability, content uniformity and tablet dissolution rate are determined in stability conditions $\left(25 \pm 2^{\circ} \mathrm{C} / 60 \pm 5 \%\right.$ relative humidity and $40 \pm 2^{\circ} \mathrm{C} / 75 \pm 5 \%$ relative humidity). These parameters were evaluated at 0 month, 3 month, 6 month and 12 month intervals. It was concluded that the coated ornidazole tablets should be stored in a high density polyethylene plastic bottle with silica gel at controlled room temperature $\left(25^{\circ} \mathrm{C}\right)$ or below their relative humidities and the presence of desiccant in the market package was essential.
\end{abstract}

KEYWORDS: Stability studies; compression coated ornidazole tablet; ethylcellulose; characterization of tablet; dissolution study.

\section{INTRODUCTION}

Stability of a pharmaceutical dosage form is defined as the time lapse during which the drug product retains, within specified limits and throughout its period of storage and use, same properties and characteristics that it possessed at the time of its manufacture. USP defines stability of pharmaceutical product as, "extent to which a product retains within specified limits and throughout its period of storage and use. It is a routine performed at various stages of product development and it must be demonstrated that pharmaceutical product characteristics from the production haven't changed until the patient's use. So, the stability is the most important quality indicator [[1]-[3]].

The stability of product is expressed as the expiry period or technically as shelf life which is valuable quality attribute for all pharmaceutical dosage forms [[1]]. It is important for patient's safety, drug efficacy, build in quality and expiration date of the product. These studies are performed by exposing the representative sample of pharmaceutical product to environmental factors such as temperature, humidity and light [[3], [5]]. Stable tablets retain their original size, shape, weight, roughness, colour variation, cracking under normal handling and storage conditions throughout their shelf life.

In general, a dosage form should be evaluated under storage conditions (with appropriate tolerances) that test its thermal stability and, if applicable, its sensitivity to moisture. The storage conditions and the lengths of studies chosen should be sufficient to cover storage, shipment, and subsequent use. The long term testing should cover a minimum of 12 months' duration on at least three primary batches at the time of submission and should be continued for a period of time sufficient to cover the proposed re-test period [[6], [7]].

The aim of this study was to evaluate the stability of compression coated ornidazole tablets as per ICH guidelines [[8], [9]]. For the preparation of tablets, pectin was used as the coating layer and mixed with ethyl cellulose to enhance the mechanical and release properties. The stability of tablets were evaluated with the parameters of weight variation, diameter, thickness, hardness, friability, content uniformity and dissolution at different time intervals of $0,1,3,6$ and 12 months as function of temperature and relative humidity.

How to cite this article: Rençber S, Şenyiğit Y, Özyazıcı M. Stability studies of compression coated ornidazole tablets for colon specific drug delivery. J Res Pharm. 2019; 23 (1): 34-43. 


\section{RESULTS AND DISCUSSION}

\subsection{Preparation of tablets}

Compression coated ornidazole tablets were successfully prepared. They showed a uniform, smooth and shiny surface, without coating defects.

\subsection{Stability studies}

Stability testing was carried out to provide evidence of how the quality of the manufactured tablets may change with time under the influence of environmental factors such as temperature and humidity and storage clousure with silicagel or without silicagel. They were important and necessary for observing drug's degradation in the process of time. Stability study was carried out in climatic chamber at $25 \pm 2^{\circ} \mathrm{C} / 60 \pm 5 \%$ relative humidity for 12 months and $40 \pm 2^{\circ} \mathrm{C} / 75 \pm 5 \%$ relative humidity for 6 months. In stability studies, compression coated ornidazole tablets were evaluated by weight variation, diameter, thickness, hardness, friability, content uniformity and tablet dissolution rate.

\subsubsection{Uniformity of weight}

In this study, at the beginning the average weights of F1-F6 were found to be $0.61 \pm 0.00 \mathrm{~g}, 0.61 \pm 0.00 \mathrm{~g}$, $0.60 \pm 0.00 \mathrm{~g}, 0.60 \pm 0.00 \mathrm{~g}, 0.60 \pm 0.00 \mathrm{~g}$ and $0.60 \pm 0.00 \mathrm{~g}$, respectively. The pharmacopoeial limit for the percentage deviation for the tablets of more than $250 \mathrm{mg}$ is $\pm 5 \%$ of the weight [[17], [17]]. At the end of stability studies, the average weight of all tablet formulations was found to be within the pharmacopeia limitations, and hence all formulations passed the test for uniformity of weight as per official requirements (Table 2). Also, the results showed at the end of 12 months the average weight of tablets in a high density polyethylene plastic bottle without silica gel increased due to relative humidity but the increase in weight is not significantly different $(\mathrm{p} \geq 0.05)$. However, no visible changes were observed in the tablets after storage in a high density polyethylene plastic bottle with silica gel for 6 / 12 months.

\subsubsection{Diameter and thickness studies}

The diameter and thickness values of all tablets in a high density polyethylene plastic bottle without silica gel were increased (Table 1). No visible changes were observed in the tablets after storage in a high density polyethylene plastic bottle with silica gel for 12 months (Table 2). In pharmacopoeias, there is no records about the diameter/thickness ratio of the tablets, however it is a general relationship that generally thickness should not be more than $1 / 2$ of the tablet diameters. According to Güven [[16]], diameter / thickness ratio must be four. In the literature, it was seen that there were tablets which did not have diameter / thickness ratio as four but nothing clear about what could be the harmful. Diameter/thickness ratio results of the tablets were presented in Table 1 and 2 and these ratios of all tablet formulations were below 4 during stability studies.

\subsubsection{Tablet hardness}

Tablet requires a certain amount of strength or hardness and resistance to friability to withstand mechanical shocks of handling in manufacture, packaging and shipping. The force required to break a tablet along its diameter is defined as the hardness or crushing strength of a tablet [[19]]. The recommended value for oral tablet hardness is 4-8 $\mathrm{kg}$ [[20], [21], [22]]. At the beginning, all of the formulations showed a high hardness value in the range of 7.23-8.17 kg/Monsanto (Table 1-2). After keeping in stability chamber for 12 months at controlled temperature and humidity, hardness values of tablets in a high density polyethylene plastic bottle without silica gel were changed significantly. However, no visible changes were observed in the tablets after storage in high density polyethylene plastic bottle with silica gel for 12 months (Table 2). Tablet hardness is not an absolute indicator of strength. Therefore, the ability of the compressed tablet to avoid fracture and breaking was also tested with friability studies.

\subsubsection{Friability}

Tablets are constantly subjected to mechanical shocks and aberration during the manufacturing, packing and transportation process. Therefore, tablets should be formulated to withstand such stress. In order to see the resistance of tablets, friability tests are routinely performed. Friability refers the ability of the compressed tablet to avoid fracture and breaking during transport and it is defined as the \% weight loss of tablets during the test. Shafer et. al. [[23]], mentioned that a loss was not more than $1 \%$ was normal but especially less than $0.8 \%$ of loss was also considered as normal. In the present study, friability values of all tablet formulations were below $1 \%$, indicating that the friability is within the limits $(p \geq 0.05)$ (Table 2$)$. 


\subsubsection{Content uniformity}

At the beginning, coated tablets were found to contain $94-97 \%$ of amount indicating uniformity of content uniformity (Table 1 and 2). The results showed that at the end of 12 months the content of tablets in a high density polyethylene plastic bottle with silica gel are not significantly different $(\mathrm{p} \geq 0.05)$ (Table 2). However, the content uniformity values were decreased in a high density polyethylene plastic bottle without silica gel slightly.

\subsubsection{In vitro drug release study}

In vitro release studies were performed to evaluate the ability of the coat layer to remain intact and prevent drug release in the physiological environment of stomach and small intestine. Therefore, conditions mimicking mouth to colon transit were used and tablets were tested in $\mathrm{pH} 1.2 \mathrm{HCl}$ buffer for $2 \mathrm{~h}$ and then the dissolution medium was replaced with $\mathrm{pH} 6.8$ phosphate buffer for $3 \mathrm{~h}$. After that, pectinolytic enzymes (Pectinex 3XL) were added to dissolution medium to evaluate the susceptibility of the coat layer to bacterial enzymatic break down in colon. These pectinolytic enzymes were good for degrading soluble and insoluble pectins with varying degrees of esterification [14, [24]].

The release rate of ornidazole was found to be $\approx 89-96 \%$ in the mimicking conditions of colon at the end of $24 \mathrm{~h}$ (Table 3-4). However, in both of the stability conditions, drug release rate of tablets in a high density polyethylene plastic bottle without silica gel decreased significantly in 24 hours at the end of 6/12 months (Table 3). However, no visible changes were observed in the tablets after storage a high density polyethylene plastic bottle with silica gel for 12 months (Figure 1, Table 4). Similar results were found in the literature for in vitro drug release of tablets ([2], [25]).

Table 1. Summary of stability studies in a high density polyethylene plastic bottle without silica gel

\begin{tabular}{|c|c|c|c|c|c|c|c|c|}
\hline Code & $\begin{array}{c}\text { Time } \\
\text { (month) }\end{array}$ & Appearance & $\begin{array}{c}\text { Weight } \\
\text { (mg) }\end{array}$ & $\begin{array}{c}\text { Diameter/ } \\
\text { thickness } \\
\text { ratio }\end{array}$ & $\begin{array}{c}\text { Hardness } \\
\text { (kg } \\
\text { /monsanto) }\end{array}$ & $\begin{array}{c}\text { Friability } \\
(\%)\end{array}$ & $\begin{array}{c}\text { Content } \\
\text { Uniformity }\end{array}$ & $\begin{array}{c}\text { Drug } \\
\text { Released } \\
(\%)(24 \mathrm{~h})\end{array}$ \\
\hline \multirow[t]{3}{*}{ F1 } & $t=0$ & Appropriate & $0.61 \pm 0.00$ & $3.61 \pm 0.03$ & $7.83 \pm 0.83$ & 0.12 & $96.32 \pm 0.29$ & $94.91 \pm 0.54$ \\
\hline & $\begin{array}{c}t=12 \\
\left(25^{\circ} \mathrm{C}\right)\end{array}$ & Appropriate & $0.64 \pm 0.02$ & $3.29 \pm 0.09$ & $7.30 \pm 1.32$ & 0.10 & $90.82 \pm 0.61$ & $85.22 \pm 0.85$ \\
\hline & $\begin{array}{c}t=6 \\
\left(40^{\circ} \mathrm{C}\right)\end{array}$ & Appropriate & $0.66 \pm 0.01$ & $3.16 \pm 0.05$ & $6.76 \pm 1.20$ & 0.08 & $85.72 \pm 0.38$ & $79.00 \pm 0.86$ \\
\hline \multirow[t]{3}{*}{ F2 } & $t=0$ & Appropriate & $0.61 \pm 0.00$ & $3.57 \pm 0.04$ & $8.17 \pm 0.29$ & 0.10 & $95.23 \pm 0.34$ & $89.50 \pm 0.64$ \\
\hline & $\begin{array}{c}t=12 \\
\left(25^{\circ} \mathrm{C}\right)\end{array}$ & Appropriate & $0.64 \pm 0.01$ & $3.07 \pm 0.06$ & $7.96 \pm 1.03$ & 0.08 & $86.28 \pm 0.35$ & $80.56 \pm 1.02$ \\
\hline & $\begin{array}{c}t=6 \\
\left(40^{\circ} \mathrm{C}\right)\end{array}$ & Appropriate & $0.65 \pm 0.02$ & $3.00 \pm 0.06$ & $6.20 \pm 0.90$ & 0.06 & $79.91 \pm 0.37$ & $75.12 \pm 2.90$ \\
\hline \multirow[t]{3}{*}{ F3 } & $\mathrm{t}=0$ & Appropriate & $0.60 \pm 0.00$ & $3.67 \pm 0.08$ & $7.95 \pm 0.62$ & 0.11 & $96.14 \pm 0.53$ & $94.06 \pm 0.49$ \\
\hline & $\begin{array}{c}t=12 \\
\left(25^{\circ} \mathrm{C}\right)\end{array}$ & Appropriate & $0.64 \pm 0.01$ & $3.09 \pm 0.16$ & $7.60 \pm 1.01$ & 0.08 & $85.50 \pm 0.36$ & $82.97 \pm 1.31$ \\
\hline & $\begin{array}{c}t=6 \\
\left(40^{\circ} \mathrm{C}\right)\end{array}$ & Appropriate & $0.65 \pm 0.02$ & $2.96 \pm 0.16$ & $6.70 \pm 1.03$ & 0.07 & $77.05 \pm 0.63$ & $78.00 \pm 1.93$ \\
\hline \multirow[t]{3}{*}{ F4 } & $t=0$ & Appropriate & $0.60 \pm 0.00$ & $3.65 \pm 0.10$ & $8.53 \pm 0.23$ & 0.16 & $100.0 \pm 0.56$ & $96.84 \pm 0.35$ \\
\hline & $\begin{array}{c}t=12 \\
\left(25^{\circ} \mathrm{C}\right)\end{array}$ & Appropriate & $0.63 \pm 0.01$ & $3.24 \pm 0.07$ & $7.20 \pm 1.03$ & 0.11 & $87.98 \pm 0.46$ & $87.00 \pm 1.05$ \\
\hline & $\begin{array}{l}\mathrm{t}=6 \\
\left(40^{\circ} \mathrm{C}\right)\end{array}$ & Appropriate & $0.64 \pm 0.03$ & $3.12 \pm 0.07$ & $6.30 \pm 1.00$ & 0.09 & $78.28 \pm 0.57$ & $79.25 \pm 1.99$ \\
\hline \multirow[t]{3}{*}{ F5 } & $\mathrm{t}=0$ & Appropriate & $0.60 \pm 0.00$ & $3.51 \pm 0.06$ & $7.87 \pm 0.63$ & 0.19 & $96.46 \pm 0.53$ & $91.67 \pm 1.92$ \\
\hline & $\begin{array}{c}t=12 \\
\left(25^{\circ} \mathrm{C}\right)\end{array}$ & Appropriate & $0.64 \pm 0.01$ & $3.35 \pm 0.26$ & $7.30 \pm 1.05$ & 0.15 & $82.85 \pm 0.29$ & $79.03 \pm 1.24$ \\
\hline & $\begin{array}{c}\mathrm{t}=6 \\
\left(40^{\circ} \mathrm{C}\right)\end{array}$ & Appropriate & $0.65 \pm 0.01$ & $3.32 \pm 0.26$ & $6.40 \pm 0.46$ & 0.12 & $78.01 \pm 0.38$ & $77.78 \pm 2.29$ \\
\hline \multirow[t]{3}{*}{ F6 } & $\mathrm{t}=0$ & Appropriate & $0.60 \pm 0.00$ & $3.48 \pm 0.08$ & $7.23 \pm 0.23$ & 0.13 & $94.45 \pm 0.19$ & $94.31 \pm 1.25$ \\
\hline & $\begin{array}{c}t=12 \\
\left(25^{\circ} \mathrm{C}\right)\end{array}$ & Appropriate & $0.64 \pm 0.01$ & $3.12 \pm 0.09$ & $6.60 \pm 1.30$ & 0.10 & $85.78 \pm 0.55$ & $83.72 \pm 1.56$ \\
\hline & $\begin{array}{c}t=6 \\
\left(40^{\circ} \mathrm{C}\right)\end{array}$ & Appropriate & $0.66 \pm 0.02$ & $3.07 \pm 0.09$ & $5.71 \pm 029$ & 0.08 & $72.15 \pm 0.76$ & $76.00 \pm 2.29$ \\
\hline
\end{tabular}


Table 2. Summary of stability studies in a high density polyethylene plastic bottle with silica gel .

\begin{tabular}{|c|c|c|c|c|c|c|c|c|}
\hline Code & $\begin{array}{c}\text { Time } \\
\text { (month) }\end{array}$ & Appearance & $\begin{array}{l}\text { Weight } \\
\text { (mg) }\end{array}$ & $\begin{array}{c}\text { Diameter/ } \\
\text { thickness } \\
\text { ratio }\end{array}$ & $\begin{array}{c}\text { Hardness } \\
\text { (kg } \\
\text { /monsanto) }\end{array}$ & $\begin{array}{c}\text { Friability } \\
(\%)\end{array}$ & $\begin{array}{c}\text { Content } \\
\text { Uniformity }\end{array}$ & $\begin{array}{c}\text { Drug } \\
\text { Released } \\
(\%)(24 \mathrm{~h})\end{array}$ \\
\hline \multirow[t]{3}{*}{ F1 } & $t=0$ & Appropriate & $0.61 \pm 0.00$ & $3.61 \pm 0.03$ & $7.83 \pm 0.83$ & 0.12 & $96.32 \pm 0.29$ & $94.91 \pm 0.54$ \\
\hline & $\begin{array}{c}t=12 \\
\left(25^{\circ} \mathrm{C}\right)\end{array}$ & Appropriate & $0.61 \pm 0.00$ & $3.60 \pm 0.05$ & $7.81 \pm 0.35$ & 0.12 & $96.50 \pm 0.32$ & $94.85 \pm 0.48$ \\
\hline & $\begin{array}{c}t=6 \\
\left(40^{\circ} \mathrm{C}\right)\end{array}$ & Appropriate & $0.62 \pm 0.01$ & $3.59 \pm 0.04$ & $7.79 \pm 0.42$ & 0.11 & $95.12 \pm 0.18$ & $93.21 \pm 1.74$ \\
\hline \multirow[t]{3}{*}{ F2 } & $t=0$ & Appropriate & $0.61 \pm 0.00$ & $3.57 \pm 0.04$ & $8.17 \pm 0.29$ & 0.10 & $95.23 \pm 0.34$ & $89.50 \pm 0.64$ \\
\hline & $\begin{array}{c}t=12 \\
\left(25^{\circ} \mathrm{C}\right)\end{array}$ & Appropriate & $0.61 \pm 0.00$ & $3.56 \pm 0.03$ & $8.15 \pm 0.52$ & 0.10 & $95.61 \pm 0.21$ & $89.85 \pm 0.96$ \\
\hline & $\begin{array}{c}t=6 \\
\left(40^{\circ} \mathrm{C}\right)\end{array}$ & Appropriate & $0.62 \pm 0.00$ & $3.55 \pm 0.05$ & $8.12 \pm 0.32$ & 0.09 & $94.02 \pm 0.38$ & $86.14 \pm 2.23$ \\
\hline \multirow[t]{3}{*}{ F3 } & $t=0$ & Appropriate & $0.60 \pm 0.00$ & $3.67 \pm 0.08$ & $7.95 \pm 0.62$ & 0.11 & $96.14 \pm 0.53$ & $94.06 \pm 0.49$ \\
\hline & $\begin{array}{c}t=12 \\
\left(25^{\circ} \mathrm{C}\right)\end{array}$ & Appropriate & $0.60 \pm 0.00$ & $3.67 \pm 0.06$ & $7.93 \pm 0.46$ & 0.10 & $94.41 \pm 0.45$ & $94.64 \pm 1.71$ \\
\hline & $\begin{array}{c}t=6 \\
\left(40^{\circ} \mathrm{C}\right)\end{array}$ & Appropriate & $0.61 \pm 0.00$ & $3.65 \pm 0.03$ & $7.91 \pm 0.61$ & 0.09 & $92.97 \pm 0.26$ & $91.71 \pm 1.23$ \\
\hline \multirow[t]{3}{*}{ F4 } & $t=0$ & Appropriate & $0.60 \pm 0.00$ & $3.65 \pm 0.10$ & $7.53 \pm 0.23$ & 0.16 & $96.64 \pm 0.56$ & $96.84 \pm 0.35$ \\
\hline & $\begin{array}{c}t=12 \\
\left(25^{\circ} \mathrm{C}\right)\end{array}$ & Appropriate & $0.60 \pm 0.01$ & $3.64 \pm 0.04$ & $7.50 \pm 0.35$ & 0.15 & $96.00 \pm 0.42$ & $97.42 \pm 1.26$ \\
\hline & $\begin{array}{c}t=6 \\
\left(40^{\circ} \mathrm{C}\right)\end{array}$ & Appropriate & $0.61 \pm 0.00$ & $3.62 \pm 0.08$ & $8.48 \pm 0.48$ & 0.13 & $95.24 \pm 0.51$ & $94.16 \pm 1.75$ \\
\hline \multirow[t]{3}{*}{ F5 } & $t=0$ & Appropriate & $0.60 \pm 0.00$ & $3.51 \pm 0.06$ & $7.87 \pm 0.63$ & 0.19 & $96.46 \pm 0.53$ & $91.67 \pm 1.92$ \\
\hline & $\begin{array}{c}t=12 \\
\left(25^{\circ} \mathrm{C}\right)\end{array}$ & Appropriate & $0.60 \pm 0.00$ & $3.50 \pm 0.07$ & $7.85 \pm 0.55$ & 0.18 & $96.03 \pm 0.23$ & $90.93 \pm 1.35$ \\
\hline & $\begin{array}{c}t=6 \\
\left(40^{\circ} \mathrm{C}\right)\end{array}$ & Appropriate & $0.61 \pm 0.01$ & $3.48 \pm 0.04$ & $7.81 \pm 0.62$ & 0.17 & $94.34 \pm 0.36$ & $88.04 \pm 1.69$ \\
\hline \multirow[t]{3}{*}{ F6 } & $t=0$ & Appropriate & $0.60 \pm 0.00$ & $3.48 \pm 0.08$ & $7.23 \pm 0.23$ & 0.13 & $94.45 \pm 0.19$ & $94.31 \pm 1.25$ \\
\hline & $\begin{array}{c}t=12 \\
\left(25^{\circ} \mathrm{C}\right)\end{array}$ & Appropriate & $0.60 \pm 0.01$ & $3.47 \pm 0.05$ & $7.20 \pm 0.71$ & 0.12 & $93.95 \pm 0.28$ & $93.12 \pm 1.14$ \\
\hline & $\begin{array}{c}t=6 \\
\left(40^{\circ} \mathrm{C}\right)\end{array}$ & Appropriate & $0.61 \pm 0.00$ & $3.45 \pm 0.04$ & $7.18 \pm 0.28$ & 0.11 & $92.05 \pm 0.35$ & $91.55 \pm 1.62$ \\
\hline
\end{tabular}



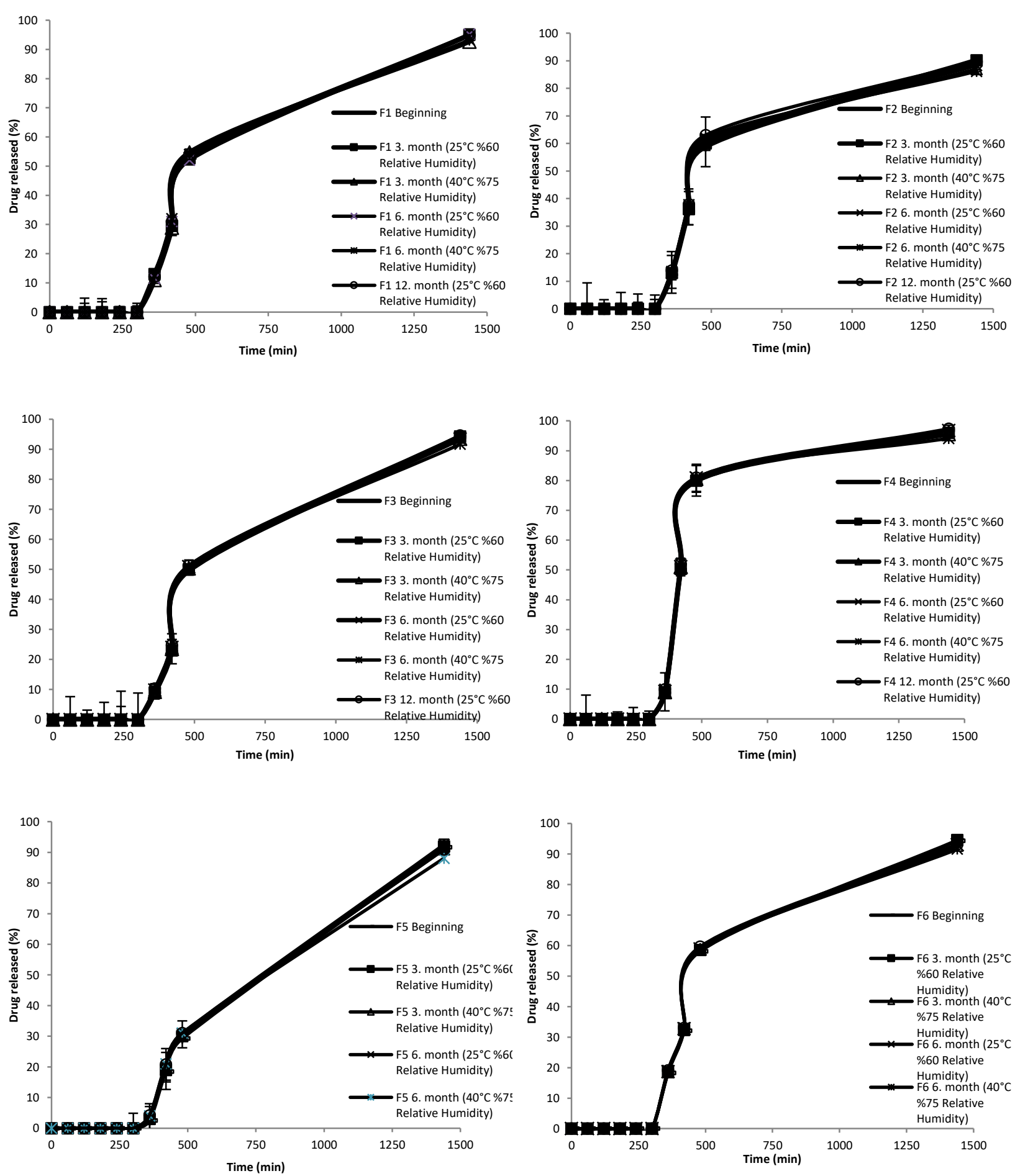

Figure 1. In vitro drug release profiles of compression coated ornidazole tablets in a high density polyethylene plastic bottle with silica gel. 
Table 3. In vitro drug release results of compression coated ornidazole tablets in a high density polyethylene plastic bottle without silica gel.

\begin{tabular}{|c|c|c|c|c|c|}
\hline \multirow{3}{*}{ Code } & \multirow{3}{*}{ Dissolution Time (hour) } & \multicolumn{4}{|c|}{ Drug Released (\%) } \\
\hline & & Beginning & 3. month & 6. month & 12. month \\
\hline & & \multicolumn{4}{|c|}{$25^{\circ} \mathrm{C} / 60 \pm 5 \%$ Relative Humidity } \\
\hline \multirow[t]{2}{*}{ F1 } & 2 & $0 \pm 0$ & $0 \pm 0$ & $0 \pm 0$ & $0 \pm 0$ \\
\hline & 24 & $94.91 \pm 0.54$ & $90.00 \pm 1.79$ & $88.21 \pm 2.76$ & $85.22 \pm 0.85$ \\
\hline \multirow[t]{2}{*}{ F2 } & 2 & $0 \pm 0$ & $0 \pm 0$ & $0 \pm 0$ & $0 \pm 0$ \\
\hline & 24 & $89.50 \pm 0.64$ & $85.44 \pm 2.76$ & $83.69 \pm 2.93$ & $80.56 \pm 1.02$ \\
\hline \multirow[t]{2}{*}{ F3 } & 2 & $0 \pm 0$ & $0 \pm 0$ & $0 \pm 0$ & $0 \pm 0$ \\
\hline & 24 & $94.06 \pm 0.49$ & $89.00 \pm 2.76$ & $86.81 \pm 2.02$ & $82.97 \pm 1.31$ \\
\hline \multirow[t]{2}{*}{ F4 } & 2 & $0 \pm 0$ & $0 \pm 0$ & $0 \pm 0$ & $0 \pm 0$ \\
\hline & 24 & $96.84 \pm 0.35$ & $92.00 \pm 2.20$ & $90.00 \pm 2.98$ & $87.00 \pm 1.05$ \\
\hline \multirow[t]{2}{*}{ F5 } & 2 & $0 \pm 0$ & $0 \pm 0$ & $0 \pm 0$ & $0 \pm 0$ \\
\hline & 24 & $91.67 \pm 1.92$ & $88.34 \pm 2.27$ & $82.22 \pm 2.66$ & $79.03 \pm 1.24$ \\
\hline \multirow[t]{3}{*}{ F6 } & 2 & $0 \pm 0$ & $0 \pm 0$ & $0 \pm 0$ & $0 \pm 0$ \\
\hline & 24 & $94.31 \pm 1.25$ & $90.45 \pm 1.15$ & $88.61 \pm 1.70$ & $83.72 \pm 1.56$ \\
\hline & & \multicolumn{4}{|c|}{$40^{\circ} \mathrm{C} / 75 \pm 5 \%$ Relative Humidity } \\
\hline \multirow[t]{2}{*}{ F1 } & 2 & $0 \pm 0$ & $0 \pm 0$ & $0 \pm 0$ & - \\
\hline & 24 & $94.91 \pm 0.54$ & $87.53 \pm 2.44$ & $79.00 \pm 0.86$ & - \\
\hline \multirow[t]{2}{*}{ F2 } & 2 & $0 \pm 0$ & $0 \pm 0$ & $0 \pm 0$ & - \\
\hline & 24 & $89.50 \pm 0.64$ & $79.20 \pm 2.31$ & $75.12 \pm 2.90$ & - \\
\hline \multirow[t]{2}{*}{ F3 } & 2 & $0 \pm 0$ & $0 \pm 0$ & $0 \pm 0$ & - \\
\hline & 24 & $94.06 \pm 0.49$ & $86.71 \pm 2.51$ & $78.00 \pm 1.93$ & - \\
\hline \multirow[t]{2}{*}{ F4 } & 2 & $0 \pm 0$ & $0 \pm 0$ & $0 \pm 0$ & - \\
\hline & 24 & $96.84 \pm 0.35$ & $89.47 \pm 1.65$ & $79.25 \pm 1.99$ & - \\
\hline \multirow[t]{2}{*}{ F5 } & 2 & $0 \pm 0$ & $0 \pm 0$ & $0 \pm 0$ & - \\
\hline & 24 & $91.67 \pm 1.92$ & $84.52 \pm 2.32$ & $77.78 \pm 2.29$ & - \\
\hline \multirow[t]{2}{*}{ F6 } & 2 & $0 \pm 0$ & $0 \pm 0$ & $0 \pm 0$ & - \\
\hline & 24 & $94.31 \pm 1.25$ & $86.00 \pm 2.49$ & $76.00 \pm 2.29$ & - \\
\hline
\end{tabular}


Table 4. In vitro drug release results of compression coated ornidazole tablets in a high density polyethylene plastic bottle with silica gel.

\begin{tabular}{|c|c|c|c|c|c|}
\hline \multirow{3}{*}{ Code } & \multirow{3}{*}{ Dissolution Time (hour) } & \multicolumn{4}{|c|}{ Drug Released (\%) } \\
\hline & & Beginning & 3. month & 6. month & 12. month \\
\hline & & \multicolumn{4}{|c|}{$25^{\circ} \mathrm{C} / 60 \pm 5 \%$ Relative Humidity } \\
\hline \multirow[t]{2}{*}{ F1 } & 2 & $0 \pm 0$ & $0 \pm 0$ & $0 \pm 0$ & $0 \pm 0$ \\
\hline & 24 & $94.91 \pm 0.54$ & $95.02 \pm 0.98$ & $95.20 \pm 2.01$ & $94.85 \pm 0.48$ \\
\hline \multirow[t]{2}{*}{ F2 } & 2 & $0 \pm 0$ & $0 \pm 0$ & $0 \pm 0$ & $0 \pm 0$ \\
\hline & 24 & $89.50 \pm 0.64$ & $90.21 \pm 1.52$ & $88.26 \pm 0.98$ & $89.85 \pm 0.96$ \\
\hline \multirow[t]{2}{*}{ F3 } & 2 & $0 \pm 0$ & $0 \pm 0$ & $0 \pm 0$ & $0 \pm 0$ \\
\hline & 24 & $94.06 \pm 0.49$ & $94.31 \pm 0.95$ & $93.45 \pm 1.19$ & $94.64 \pm 1.71$ \\
\hline \multirow[t]{2}{*}{ F4 } & 2 & $0 \pm 0$ & $0 \pm 0$ & $0 \pm 0$ & $0 \pm 0$ \\
\hline & 24 & $96.84 \pm 0.35$ & $96.03 \pm 1.55$ & $96.78 \pm 1.88$ & $97.42 \pm 1.26$ \\
\hline \multirow[t]{2}{*}{ F5 } & 2 & $0 \pm 0$ & $0 \pm 0$ & $0 \pm 0$ & $0 \pm 0$ \\
\hline & 24 & $91.67 \pm 1.92$ & $91.94 \pm 1.62$ & $92.55 \pm 1.86$ & $90.93 \pm 1.35$ \\
\hline \multirow[t]{3}{*}{ F6 } & 2 & $0 \pm 0$ & $0 \pm 0$ & $0 \pm 0$ & $0 \pm 0$ \\
\hline & 24 & $94.31 \pm 1.25$ & $94.54 \pm 1.23$ & $93.05 \pm 1.23$ & $93.12 \pm 1.14$ \\
\hline & & \multicolumn{4}{|c|}{$40^{\circ} \mathrm{C} / 75 \pm 5 \%$ Relative Humidity } \\
\hline \multirow[t]{2}{*}{ F1 } & 2 & $0 \pm 0$ & $0 \pm 0$ & $0 \pm 0$ & - \\
\hline & 24 & $94.91 \pm 0.54$ & $92.66 \pm 2.01$ & $93.21 \pm 1.74$ & - \\
\hline \multirow[t]{2}{*}{ F2 } & 2 & $0 \pm 0$ & $0 \pm 0$ & $0 \pm 0$ & - \\
\hline & 24 & $89.50 \pm 0.64$ & $87.00 \pm 1.88$ & $86.14 \pm 2.23$ & - \\
\hline \multirow[t]{2}{*}{ F3 } & 2 & $0 \pm 0$ & $0 \pm 0$ & $0 \pm 0$ & - \\
\hline & 24 & $94.06 \pm 0.49$ & $93.56 \pm 1.48$ & $91.71 \pm 1.23$ & - \\
\hline \multirow[t]{2}{*}{ F4 } & 2 & $0 \pm 0$ & $0 \pm 0$ & $0 \pm 0$ & - \\
\hline & 24 & $96.84 \pm 0.35$ & $95.61 \pm 1.34$ & $94.16 \pm 1.75$ & - \\
\hline \multirow[t]{2}{*}{ F5 } & 2 & $0 \pm 0$ & $0 \pm 0$ & $0 \pm 0$ & - \\
\hline & 24 & $91.67 \pm 1.92$ & $90.95 \pm 1.36$ & $88.04 \pm 1.69$ & - \\
\hline \multirow[t]{2}{*}{ F6 } & 2 & $0 \pm 0$ & $0 \pm 0$ & $0 \pm 0$ & - \\
\hline & 24 & $94.31 \pm 1.25$ & $92.68 \pm 1.27$ & $91.55 \pm 1.62$ & - \\
\hline
\end{tabular}

\section{CONCLUSION}

In this study, core tablets containing ornidazole were compression coated with different Pectin - EC mixtures and stability studies were performed in a high density polyethylene plastic bottle with/without silica gel under at $25 \pm 2^{\circ} \mathrm{C} / 60 \pm 5 \%$ relative humidity and $40 \pm 2^{\circ} \mathrm{C} / 75 \pm 5 \%$ relative humidity. The stored tablets were evaluated for various tablet characteristics including weight, diameter, thickness, hardness, friability, content uniformity and dissolution rate. No visible changes were observed in the tablets after storage in high density polyethylene plastic bottle with silica gel for 12 months. It was concluded that the coated ornidazole tablets should be stored in a high density polyethylene plastic bottle with silica gel at controlled room temperature $\left(25^{\circ} \mathrm{C}\right)$ or below their relative humidities and the presence of desiccant in the market package was essential. 


\section{MATERIALS AND METHODS}

\subsection{Materials}

Ornidazole was a kind gift from Abdi İbrahim İlaç San. ve Tic. A.Ş. (Turkey). Pectin was donated by Wyeth İlaçları A.Ş. (Turkey). Ethylcellulose (EC) N7, N10 and N100 were obtained from Hercules (England). Pectinex 3XL was a gift from Novo Nordisk (Switzerland). All other solvents and reagents were of analytical grade.

\subsection{Preparation of tablets}

Tablets were prepared in accordance with Özyazıcı et al's study [[10]]. Core tablets were prepared with ornidazole and polyvinylprolidone (1\%, PVP) by wet granulation method.

Core tablets containing $200 \mathrm{mg}$ ornidazole were compression coated with $400 \mathrm{mg}$ coating material. Briefly, half of the coating material was placed in the flat-faced punches. The core tablet was carefully positioned in the centre of the die cavity and was filled with the other half of the coating material. The coating material was compressed around the core using a hydraulic hand press tablet machine (Perkin Elmer) with a pressure to 5.0 tons for $10 \mathrm{~s}$ [[11], [12]]. Compositions of compression coat are given in Table 5.

Table 5. Composition of the compression coat

\begin{tabular}{cc}
\hline Formulation Code & Composition of Compression Coat \\
\hline F1 & $\% 80$ Pectin - \%20 EC N7 \\
F2 & $\% 60$ Pectin - \%40 EC N7 \\
F3 & $\% 80$ Pectin - \%20 EC N10 \\
F4 & $\% 60$ Pectin - \%40 EC N10 \\
F5 & $\% 80$ Pectin - \%20 EC N100 \\
F6 & $\% 60$ Pectin - \%40 EC N100 \\
\hline
\end{tabular}

\subsection{Stability studies}

The compression coated ornidazole tablets were submitted to stability test and they were maintained in a high density polyethylene plastic bottle with/without silica gel according to ICH guidelines at $25 \pm 2^{\circ} \mathrm{C} / 60$ $\pm 5 \%$ relative humidity for 12 months and $40 \pm 2^{\circ} \mathrm{C} / 75 \pm 5 \%$ relative humidity for 6 months [[7], [13], [14]]. Physical attributes of the tablets, i.e., appearance, uniformity of weight, diameter, thickness, hardness, friability, content uniformity and in vitro drug release profiles were tested over a period of 12 months.

\subsubsection{Uniformity of weight}

20 tablets were randomly selected and weighed individually using an electronic balance (Sartorius) and the mean weights of tablets were calculated.

\subsubsection{Diameter and thickness studies}

The diameter and thickness measurements were conducted on 3 tablets using Mitutoyo Absolute Digimatic Caliper and diameter/thickness ratio was calculated. Results are expressed as the mean and standard deviation.

\subsubsection{Tablet hardness}

Ten tablets were randomly selected and individually placed between two anvils of the hardness tester (Monsanto type) and force is applied. Then, the hardness of tablets reported in $\mathrm{kg} / \mathrm{monsanto.}$

\subsubsection{Friability test}

Friability of the tablets was determined using friabilator (Roche friabilitor). 10 tablets were weighted and subjected to the combined abrasion and shock in a plastic chamber revolving at $25 \mathrm{rpm}$ for 4 minutes. In each revolution, tablets were dropped at a height of 6 inches. Tablets were de-dusted using a brush and reweighed. The percentage of the tablets friability was calculated as Equation 1. The desirable friability was determined as lower than $1 \%$.

Friability $=\frac{\text { initial weight of tablets }- \text { final weight of tablets }}{\text { initial weight of tablets }} \times 100 \quad$ (Equation 1$)$ 


\subsubsection{Content uniformity}

For content uniformity test, tablets were pulverized, $\mathrm{pH} 6.8$ phosphate buffer was added to adjust the volume to $10 \mathrm{~mL}$ and then extracted by shaking for $1 \mathrm{~h}$. The mixture was filtered and the drug was assayed spectrophotometrically at $319 \mathrm{~nm}$ [[15]].

\subsubsection{In vitro drug release study}

In vitro drug release studies were performed using paddle method according to USP 34 and $900 \mathrm{~mL}$ medium was maintained at $37^{\circ} \mathrm{C}$. Tablets were tested for drug release for $2 \mathrm{~h}$ in $\mathrm{pH} 1.2 \mathrm{HCl}$ buffer as the average gastric emptying time is about $2 \mathrm{~h}$. Then, the dissolution medium was replaced with $\mathrm{pH} 6.8$ phosphate buffer and drug release was tested for $3 \mathrm{~h}$ as the average small intestinal transit time is about $3 \mathrm{~h}$. At the end of these time periods, $1 \%$ pectinolytic enzyme was added to dissolution medium to simulate colonic transit and the study continued up to $24 \mathrm{~h}$. At various time intervals, samples were withdrawn and the amount of ornidazole released from was estimated spectrophotometrically (Shimadzu, UV 1208). The $\lambda_{\max }$ values were $277 \mathrm{~nm}$ for $\mathrm{pH} 1.2 \mathrm{HCl}$ buffer and $319 \mathrm{~nm}$ for $\mathrm{pH} 6.8$ phosphate buffer, respectively [[10]].

\subsubsection{Statistical data analysis}

Statistical data analysis was performed using the Student's t-test with $\mathrm{P}<0.05$ as the minimal level of significance.

Acknowledgments: This study was supported by a research grant from Ege University (03/ECZ/006 and 04/ECZ/028).

Author contributions: Conception - S.R., Z.Ş., M.Ö.; Design - S.R., Z.Ş., M.Ö.; Supervision - S.R., Z.Ş., M.Ö.; Resources - S.R., Z.Ş., M.Ö.; Materials - S.R., Z.Ş., M.Ö.; Data Collection and/or Processing - S.R., Z.Ş., M.Ö.; Analysis and/or Interpretation - S.R., Z.Ş., M.Ö.; Literature Search -S.R., Z.Ş., M.Ö.; Writing Manuscript-S.R., Z.Ş., M.Ö.; Critical Review - S.R., Z.Ş., M.Ö.

Conflict of interest statement: The authors report no conflicts of interest. The authors alone are responsible for the content and writing of the paper.

\section{REFERENCES}

[1] Punam T, Shubhangi W, Rahul S, Sargar A, Bhujbal A, Shinde A. Stability study of dosage form: An inovative step. World J Pharm Pharm Sci. 2014; 3(2): 1031-1050.

[2] Bozkır A, Coşkun Cetintas H, Saka O M. Investigation of the stability with bracketing design in tablet form. Pharm Anal Acta. 2013; S1-005. [CrossRef]

[3] Acartürk F, Agabeyoglu I, Celebi N, Degim T, Degim Z, Doğanay T, Takka S, Tırnaksız F. Reaksiyon kinetiği ve stabilite. In: Modern Farmasötik Teknoloji. Türk Eczacıları Birliği Eczacılık Akademisi Yayını, Ankara, 2007, No:1, pp. 141-182. ISBN:978-975-01139-1-8.

[4] Bajaj S, Singla D, Sakhuja N. Stability testing of pharmaceutical products. J Appl Pharm Sci. 2012; 2(3): 129-138. [CrossRef]

[5] Al-Gohary OMN, Al-Kassas RS. Stability studies of aspirin-magaldrate double layer tablets. Pharm Acta Helv. 2000; 74(4): 351-360. [CrossRef]

[6] Note for guidance on stability testing: Stability testing of new drug substances and products. In: ICH Topic Q 1 A (R2) Stability Testing of new Drug Substances and Products. European Medicines Agency. 2003; $\mathrm{CPMP} / \mathrm{ICH} / 2736 / 99$

[7] SADC Guideline For Stability Testing, 2004.

[8] Krishnaiah YSR, Muzib YI, Rao GS, Bhaskar P, Satyanarayana V. Studies on the development of colon targeted oral drug delivery systems for ornidazole in the treatment of amoebiasis. Drug Deliv. 2003; 10: 111-117. [CrossRef]

[9] World Health Organization, Stability testing of active pharmaceutical ingredients and finished pharmaceutical products. WHO Technical Report Series, 2009; No. 953.

[10] Özyazıcı M, Ay Şenyiğit Z, Aksu B. Compression coated ornidazole tablets for colon spesific drug delivery: Development, in vitro evaluation and optimisation by artificial neural network. Lat Am J Pharm. 2015; 34 (4): 681-93. 
[11] Krishnaiah YSR, Satyanarayana S, Rama Prasad YV, Narasimha Rao S. Evaluation of guar gum as a compression coat for drug targeting to colon. Int J Pharm. 1998; 171: 137-146. [CrossRef]

[12] Türkoğlu M, Uğurlu T. In vitro evaluation of Pectin-HPMC compression coated 5-aminosalicylic acid tablets for colonic delivery. Eur J Pharm Biopharm. 2002; 53: 65-73. [CrossRef]

[13] Stability testing of active pharmaceutical ingredients and finished pharmaceutical products, World Health Organization, Technical Report Series, No. 953, 2009.

[14] Ren Y, Jiang L, Yang S, Gao S, Yu H, Hu J, Hu D, Mao W, Peng H, Zhou Y. Design and preparation of a novel colontargeted tablet of hydrocortisone. Braz J Pharm Sci. 2016; 52(2): e15009. [CrossRef]

[15] Baloğlu E, Hizarcıoğlu SY. Quality control studies on enalapril maleate tablets available on the Turkish drug market. Ankara Ecz Fak Derg. 2001; 30(1): 1-16. [CrossRef]

[16] Güven KC. İlaç Endüstrisi Teknolojisi. Cilt II, Hüsnütabiat Matbaası, İstanbul, 1979.

[17] Aslani A, Jahangiri H. Formulation, Characterization and physicochemical evaluation of ranitidine effervescent tablets. Adv Pharm Bull. 2013; 3(2): 315-322. [CrossRef]

[18] Potu A, Pasunooti S, Veerareddy P, Burra S. Formulation and evaluation of fenoprofen calcium compressed coated tablets for colon specific drug delivery. Asian J Pharm Clin Res. 2011; 4(2): 88-95.

[19] Carstensen JT. Drug stability principles and practices. Marcel Dekker Inc., New York, 1990, pp. 209-261.

[20] Parrot EL. Solid pharmaceuticals, In: Pharmaceutical Technology, 3rd ed., Burgess Publishing Company, USA, 1971, pp. 58-106.

[21] Geçgil Ş. Katı İlaç Şekilleri, In: Farmasötik Teknolojiye Başlangıç, Cihan Matbaacılık, İstanbul, 1991, pp. 365.

[22] Özyazıcı M, Sevgi F. Formulation and evaluation of salbutamol sulphate tablets prepared by direct compression using various excipients: Statistical comparison of dissolution methods and tablet properties. FABAD J Pharm Sci. 2003; $28: 1-11$.

[23] Shafer E, Wollish EG, Engel CE. The Roche Friabilator. J Am Pharm Assoc Sci. 1956; 45: 116-144.

[24] Novozymes product sheet, fruit \& Vegetable/2002-15554-02.pdf, (2008) Available at <www.novozymes.com>.

[25] Faisal U, Ibrahim J, Rasheed S, Ranjha NM, Hussain L, Massud A. Accelerated stability studies of flurbiprofen film coated tablets of five different national brands in Pakistan. J Drug Deliv Ther. 2013; 3 (2): 9-11. [CrossRef]

This is an open access article which is publicly available on our journal's website under Institutional Repository at http://dspace.marmara.edu.tr. 